\title{
Hemophilus influenzae pericarditis in an adult
}

\author{
GRANT M. BARNUM, CAPT, USAF, MC \\ TERRANCE O. KURTZ, DO \\ GARY L. HOFF, DO
}

\begin{abstract}
Purulent pericarditis carries a poor prognosis, partly because diagnosis is difficult early in the course of the disease. Pericarditis in adults rarely is caused by Hemophilus influenzae. Literature review indicates that adult pericarditis caused by this organism is less likely to be fatal than when other bacterial agents are implicated. In the reported case of $H$ influenzae pericarditis in a 43-year-old man, the correct diagnosis was not made until shortly before death. Suppression of fever by anti-inflammatory medication may have obscured the fact that the infective agent was bacterial rather than viral. The practice of delaying pericardiocentesis and administering nonsteroidal, antiinflammatory agents in presumed viral pericarditis may need to be reconsidered.
\end{abstract}

In adults, purulent pericarditis only rarely is caused by Hemophilus influenzae; our literature review yielded just 17 cases. ${ }^{1-14}$ Purulent pericarditis is difficult to diagnose, and failure to institute early, adequate treatment can have grave consequences.

A case of $H$ influenzae pericarditis with bacteremia but no other clinical site of infection is presented. Standard treatment for presumed viral pericarditis may have delayed accurate diagnosis and institution of appropriate therapy.

\section{Report of case \\ Preadmission history}

A 43-year-old man developed flu-like symptoms (fever, cough, myalgias, arthralgias, headache, nausea, and diarrhea) $2^{1 / 2}$ weeks before admission. Around the same time, other family members were ill with similar symptoms.

Three days into his illness, the patient's initial symptoms were improving, but he began to experience a constant, burning substernal discomfort, with radiation to the left arm. The pain was associated with dyspnea, and it was worsened by coughing and deep breathing. It was not worsened by exertion or influenced by position.

Ten days before admission, the pain became so severe that he went to his primary physician. An ECG revealed marked ST-segment elevation in leads V3 and V4 and left axis deviation. The primary diagnosis was myocardial infarction, and he was admitted to his local hospital. He continued to experience pain, but only intermittently. These episodes responded poorly to nitroglycerin, and after three days he was transferred to a tertiary medical center for further evaluation. Chest roentgenography at that institution demonstrated that the heart size and lung fields were normal. Coronary angiography showed normal coronary arteries and normal left ventricular function. It was concluded that the patient probably had a viral illness, possibly with pleuritis and pericarditis, which would account for the ECG changes. Indomethacin therapy ( $50 \mathrm{mg}$ three times a day) was instituted, and he was released.

The chest discomfort was relieved partially for a few days, but on the sixth day postangiography, he returned to his hometown hospital with severe chest pain. The liver enzyme values were found to be elevated, and he was transferred to our institution.

\section{Hospital course}

Despite the $150 \mathrm{mg}$ of indomethacin and up to $9 \mathrm{~g}$ of acetaminophen per day prescribed at his local hospital, at admission the patient was experiencing significant burning, substernal pain, with radiation to the left shoulder and arm. He also complained of cephalgia, nausea, and marked dyspnea, despite oxygen administration via nasal cannula.

The past medical history was significant for hepatitis at age 12 and multiple peptic ulcers, for which the patient underwent pyloroplasty and vagotomy at age 28 . $\mathrm{He}$ occasionally consumed large quantities of ethanol but declined to state a specific amount, and he also had a 40 pack-year smoking history. The family history was unremarkable.

On physical examination, the patient was alert, oriented, and in considerable distress. The blood pressure was $94 / 80 \mathrm{~mm} \mathrm{Hg}$ in the right arm and $102 / 80 \mathrm{~mm} \mathrm{Hg}$ in the left arm. The heart rate was 148 beats per minute, the respiratory rate was 34 breaths per minute, and the temperature was $37^{\circ} \mathrm{C}$.

Skin and sclera were mildly icteric. Pupils were equal, round, and reactive to light and accommodation. Extraocu- 
lar muscle movements were intact. The ears, nose, mouth, and throat all were unremarkable. There was no cervical lymphadenopathy or thyroid abnormality.

The chest expanded symmetrically on inspiration, but there were occasional crackles in the left lower lung field. Variation in systolic blood pressure on respiration was less than $10 \mathrm{~mm} \mathrm{Hg}$. The second heart sound was louder than the first, with normal splitting of the second heart sound. A two-component friction rub was auscultated at the lower left sternal border; this was loudest during inspiration. There were no audible murmurs or third or fourth heart sounds.

The liver margin was tender and palpable at the right costal border and spanned $8 \mathrm{~cm}$ to percussion at the midclavicular line on the right. Bowel sounds were of normal intensity and frequency. There was mild tympany, but no masses or splenomegaly, and the abdomen was soft. Rectal examination was unremarkable, the stool was negative for occult blood. Neurologic examination of cranial nerves, muscle strength, and sharp and light touch showed normal reactions, and there were appropriate, bilaterally symmetric deep-tendon relexes.

Laboratory data obtained within the first 24 hours of admission were as follows: WBC, $27,400 / \mathrm{cu} \mathrm{mm}$; differential count, $89 \%$ segmented neutrophils, $10 \%$ lymphocytes, and $1 \%$ monocytes; and platelet count, 497,000 / cu $\mathrm{mm}$. Hemoglobin and hematocrit values were 13.4 $\mathrm{g} / \mathrm{dL}$ and $42.3 \%$, respectively. The total bilirubin value was normal at $0.8 \mathrm{mg} / \mathrm{dL}$, but liver enzyme levels were elevated (aspartate aminotransferase, 880 units $/ \mathrm{L}$; alanine aminotransferase, 590 units/L; alkaline phosphatase, 238 units $/ \mathrm{L} ; \mu$-glutamyl transpeptidase, 144 units $/ \mathrm{L}$; and lactic dehydrogenase, 1,130 units $/ \mathrm{L}$ ). The serum albumin value was only $2.15 \mathrm{~g} / \mathrm{dL}$, and cholesterol and total protein levels were depressed slightly. As for arterial blood gas values, $\mathrm{pH}$ was $7.47, \mathrm{pCO}_{2}$ was $30.5 \mathrm{~mm} \mathrm{Hg}$, and $\mathrm{pO}_{2}$ was $65 \mathrm{~mm} \mathrm{Hg}$ on room air. Other laboratory values included; sodium $141 \mathrm{mEq} / \mathrm{L}$; potassium, $4.8 \mathrm{mEq} / \mathrm{L}$; chloride, $99 \mathrm{mEq} / \mathrm{L}$; serum carbon dioxide, $32 \mathrm{mEq} / \mathrm{L}$; BUN, $23 \mathrm{mg} / \mathrm{dL}$; and serum creatinine, $1.3 \mathrm{mg} / \mathrm{dL}$. Serum glucose, calcium thyroxine, uric acid, amylase, and lipase values were unremarkable. The prothrombin and partial thromboplastin times were normal.

Admission chest roentgenography was significant for marked cardiomegaly and a small pleural effusion with mild bilateral pulmonary edema. A 12-lead ECG showed low voltage throughout all leads, sinus tachycardia, and ST-segment elevation across most leads. Two-dimensional and M-mode echocardiography revealed moderate anterior and posterior pericardial effusion without evidence of loculations but was otherwise unremarkable. The patient initially was treated with analgesics and anti-inflammatory drugs for presumed viral pericarditis (nalbuphine, $5 \mathrm{mg}$ intramuscular every 4 to 6 hours; enteric-coated aspirin, 10 grains every 4 hours, both on an as needed basis. A viral hepatitis profile was ordered. The acetaminophen was discontinued on admission because of elevated liver function tests. On the second day of hospitalization, he reported less chest pain, and his heart rate decreased to 110 beats per minute. Serial liver enzyme values gradually improved. Liver and spleen scanning showed hepatomegaly (but not splenomegaly) and increased vertebral uptake, which is evidence of hepatocellular disease. Initial blood cultures were negative.

On the fifth hospital day, the patients' temperature rose to $38.1^{\circ} \mathrm{C}$, but it returned to normal within eight hours. The cold agglutination titer was $1: 16$, and the cytomegalovirus titer was $<1: 8$. On the following day, the WBC count rose to $44,400 / \mathrm{cu} \mathrm{mm}$. Blood culturing was repeated, and blood for leptospirosis and Coxsackie virus $\mathrm{A}$ and $\mathrm{B}$ titer determinations was drawn. On the seventh day, the WBC count declined to $35,800 / \mathrm{cu} \mathrm{mm}$. The patient remained afebrile, with only mild chest discomfort, and he continued to lack a paradoxic pulse.

On the 11th day of hopitalization, the patient's blood pressure fell precipitously, although his heart rate remained at 110 beats per minute. Two of the three blood cultures from day 6 became positive for $H$ influenzae, and the preliminary report on the IgM antibody against hepatitis A was positive. The patient was transferred to the intensive care unit, blood cultures were again performed, and cefotaxime and ampicillin were administered intravenously.

During attempted right-sided heart catheterization, the patient suffered cardiopulmonary arrest, which responded to endotracheal intubation, respiratory assistance, closed-chest cardiac massage, and intravenous pressors and atropine. Bedside subxyphoid pericardiocentesis during resuscitation produced green-tinged, purulent material. An emergency pericardial window was created, and 400 to $500 \mathrm{cc}$ of thick, purulent fluid were evacuated.

Despite the return of sinus tachycardia, relief of the pericardial effusion, and intravenous pressor administration, the systolic blood pressure remained below $70 \mathrm{~mm}$ $\mathrm{Hg}$. An intra-aortic counterpulsation balloon was inserted percutaneously, which raised the systolic pressure to $90 \mathrm{~mm} \mathrm{Hg}$. However, during the next 36 hours, the patient required huge doses of intravenous fluids, pressors, and antidysrhythmic agents to maintain blood pressure and sinus rhythm. Mechanical ventilation with $100 \%$ oxygen resulted in barely adequate blood oxygenation. The blood pressure finally could no longer be maintained, and he died on the morning of the 13th hospital day.

\section{Pathologic findings}

Cultures of the pericaridal fluid proved to contain $\beta$ lactamase-negative $H$ influenzae. Upper respiratory cultures showed only normal flora. The endotracheal tube aspirate culture was negative. The findings for the Coxsackie virus $A$ and $B$ titers, febrile agglutinins, hepatitis B surface antigen, and antihepatitis B core antibody were insignificant or negative. The IgM antihepatitis A antibody test, which initially had been reported positive, was negative on the final confirmatory report.

The autopsy revealed suppurative pericarditis, with a fibrinous exudate coating both pericardial surfaces (Fig 1). The lungs contained multiple, small pulmonary em- 
boli and hyaline-like alveolar membranes. Anasarca and central hemorrhagic necrosis of the liver also were present.

\section{Discussion}

$H$ influenzae is an uncommon cause of bacterial pericarditis in adults. Of the 17 cases $^{1-14}$ found in an extensive literature search, nine involved pneumonia or empyema as well. All but two of the other eight patients had evidence of an extrapericardial site of involvement (primarily the oropharynx), with one case each of thyroiditis, ${ }^{4}$ epiglottitis, ${ }^{13}$ and meningitis. ${ }^{14}$ In our case, we were unable to find a physical sign of infection in an extrapericardial location (aside from the liver), although presumably the upper respiratory tract was the portal of entry.

All but three ${ }^{7,9}$ of the 17 patients reviewed were treated with pericardial drainage procedures. Only two $0^{1,7}$ of the 17 died, and one ${ }^{1}$ of these deaths more likely was caused by complications of the antibiotic used than by the pericarditis. Unless a significant number of cases of death from $H$ influenzae pericarditis have gone unreported, isolation of this bacterial agent from the pericardium seemingly represents a relatively good prognosis. This is surprising, because the diagnosis of purulent pericarditis in general was associated with death in more than $75 \%$ of patients in one study. ${ }^{5}$

The poor prognosis of purulent pericarditis at least in part is due to diagnostic failure. ${ }^{15}$ The illness usually is acute, with symptoms of high, spiking fevers, chills, night sweats, and dyspnea. Tachycardia is a constant feature, and a friction rub often is heard. A fall in systolic blood pressure $>10 \mathrm{~mm} \mathrm{Hg}$ on inspiration (pulsus paradoxus) and jugular venous distension (central venous pressure $>10 \mathrm{~cm}$ of water) are signs of pericardial tamponade and require drainage. ${ }^{5}$ Usually present are leukocytosis $>12,000 / \mathrm{cu} \mathrm{mm}$ with a leftward shift, cardiomegaly on the chest roentgenogram, and STsegment elevation (concave upward) on the ECG.

First-degree atrioventricular block, atrioventricular dissociation, and bundle-branch block may result from endocarditis, with extension through the valve ring as a source for the pericarditis. ${ }^{5,16}$ If right-sided heart catheterization is performed, the right atrial, right ventricular diastolic, pulmonary diastolic, and pulmonary capillary wedge pressures will be elevated; in the case of tamponade, they will be nearly equal. ${ }^{16}$

Our patient presented with the regular signs and symptoms of viral pericarditis. The typical prodrome of upper respiratory "cold" symptoms was followed by the onset of acute, severe, unremitting

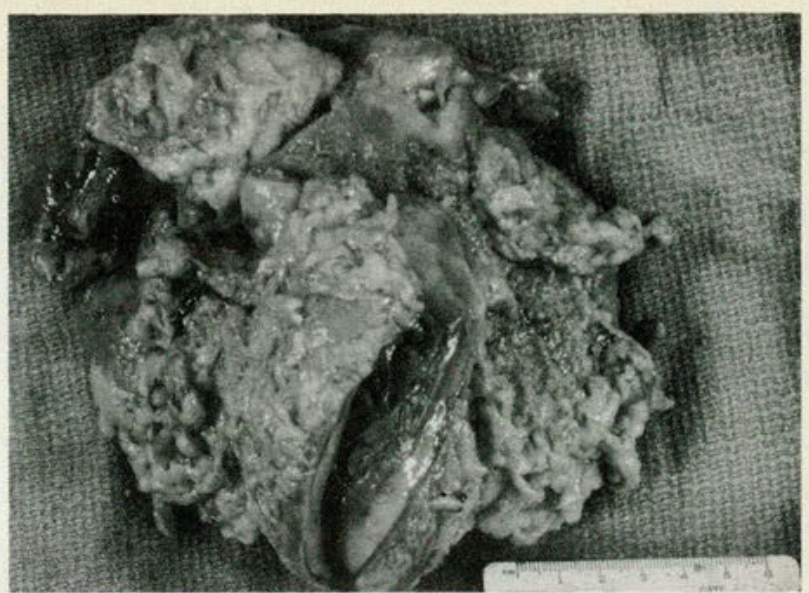

Fig 1. Visceral pericardial surface is coated with shaggy, fibrinous exudate.

chest pain. There were a pericardial friction rub and echocardiographically documented pericardial effusion.

Viral or idiopathic pericarditis usually is selflimited over one to three weeks, ${ }^{16}$ and pericardiocentesis is not required unless evidence of tamponade occurs, purulent pericarditis is suspected, or the illness fails to respond spontaneously. ${ }^{17}$ Our patient was monitored for marked jugular venous distention, hypotension, paradoxic pulse, and fever. A low-grade febrile episode did occur, but it was short-lived and resolved without antibiotics. WBC elevation persisted, but this was thought to be consistent with pericarditis and hepatitis A. (As noted previously, the preliminary IgM antihepatitis A antibody report was positive, while the postmortem report was negative.) Marked hypotension was the first sign of the gravity of the illness. This was more likely a sign of sepsis than of tamponade, because marked central venous pressure elevation and pulsus paradoxus were not evident prior to the hypotensive episode.

Our patient was treated with indomethacin throughout most of his illness. Nonsteroidal, antiinflammatory drugs have been used commonly for the treatment of pain and inflammation in pericarditis associated with trauma, myocardial infarction, and viral illness ${ }^{18}$ and in uremic pericarditis. ${ }^{19}$

In a prospective double-blinded study ${ }^{19}$ of uremic pericarditis, 11 patients were treated with indomethacin and 13 patients were given placebo. There was prompt defervescence in the patients treated with indomethacin. However, with respect to chest pain, friction rub, pericardial effusion, and the need for surgical intervention, there was no 
advantage to the use of indomethacin. If the same is true for other types of pericarditis, indomethacin may have suppressed the high, spiking fevers typical of bacterial pericarditis in our patient, without providing a significant benefit. Thus, the correct diagnosis may have been delayed critically.

\section{Conclusions}

The course of our patient's illness was unusual in that $H$ influenzae is a rare cause of purulent pericarditis in adults, there was no evident concomitant extrapericardial locus of infection, there were no high, spiking fevers, and the outcome was fatal.

Suppression of fever by indomethacin may have delayed the diagnostic pericardiocentesis and therapeutic pericardiotomy. Whether indomethacin provides a benefit other than antipyresis in pericarditis of unproved but suspected viral or idiopathic etiology needs further study. Additionally, exclusion of purulent pericarditis before institution of autoinflammatory medications may be advisable. Also, the practice of delaying pericardiocentesis in presumed viral pericarditis may need to be reconsidered.

1. Hensler VL: Influenzabazillen-perikarditis, Cardiologia 1955;27:154165.

2. Crossley K, Bigos T, Joffe CD: Hemophilus influenzae pericarditis. Am Heart $J$ 1973;85:246-251.

3. Duke M, Donovan TJ: Hemophilus influenzae pericarditis with cardiac tamponade. Am J Cardiol 1973;31:778-780.

4. Alsever RM, Stiver HG, Dinerman N, et al: Hemophilus influenzae pericarditis and empyema and thyroiditis in an adult. JAMA 1974;230:1426-1427.

5. Rubin RH, Moellering RC Jr: Clinical, microbiological, and therapeutic aspects of purulent pericarditis. Am J Med 1975;59:68-78.
6. Jafari N, Ikeda S, Taylor W, et al: Adult Hemophilus influenzae pericarditis. Del Med J 1976;48:513-515.

7. Sheikh MU, Lee WR, Koh DS: Serial echographic development of apparent hypertrophic cardiomyopathy with biventricular outflow obstruction: Documentation in Hemophilus influenzae pericarditis during hypovolemia. Am Heart J 1981;102:1069-1071.

8. Sink JD, Spray TL, Rankin JS: Hemophilus influenzae pericarditis. Clin Cardiol 1982;5:547-549.

9. Graham BS, Reiss TF, Gregory DW: Pericarditis associated with $\mathrm{He}$ mophilus influenzae type B pneumonia and bacteremia in two adults. Chest 1983;84:48-50.

10. Jennings HS III, Eskind JB, Savage AM, et al: Hemophilus influenzae purulent pericarditis with cardiac tamponade. South Med $J$ $1983 ; 76: 812-813$.

11. Buckingham TA, Wilner G, Sugar SJ: Hemophilus influenzae pericarditis in adults. Arch Intern Med 1983;143:1809-1810.

12. Kiefaber RW, Bach RD, McDowell JA: Nonsurgical treatment of Hemophilus influenzae pericarditis in an adult. Am Heart $J$ 1984;108:168169 .

13. Mier A, Shanson DC: Ampicillin-resistant Hemophilus influenzae epiglottitis and pericarditis in an adult, letter. Lancet 1984;2:817.

14. Simon HB, Southwick FS, Moellering RC Jr, et al: Hemophilus influenzae in hopitalized adults: Current perspectives. Am J Med 1980;69:219-226.

15. Klacsmann PG, Bulkley BH, Hutchins GM: The changed spectrum of purulent pericarditis. Am J Med 1977;63:666-673.

16. Lorell, BH, Braunwald E: Pericardial disease, in Braunwald E (ed.): Heart Disease: A Textbook of Cardiovascular Medicine. Philadelphia, WB Saunders Co, 1984, pp 1470-1527.

17. Permanyer-Miralda G, Sagrista-Sauleda J, Soler-Soler J: Primary acute pericardial disease: A prospective series of 231 consecutive patients. Am J Cardiol 1985;56:623-630.

18. McGinn JT, Rosati M, McGinn TG: Indomethacin in treatment of pericarditis. NY State J Med 1970;70:1783-1788.

19. Spector D, Alfred H, Siedlecki M, et al: A controlled study of the effect of indomethacin in uremic pericarditis. Kidney Int 1983;24:663669.

From the Department of Internal Medicine, Des Moines General Osteopathic Hospital, Des Moines.

Reprint requests to Dr Barnum, 1048 Jewel Pl, NE, Albuquerque, 87123. 